\title{
Analysis of Pyelonephritis-Associated Beta Hemolytic Streptococcus in Japan
}

\author{
Masaaki Minami ${ }^{*}$, Hideki Nishiyama², Shinoba Ikegami², Takuya Hattori², Makoto Kawashima ${ }^{3}$, \\ Michio Ohta ${ }^{4}$
}

\author{
${ }^{1}$ Department of Bacteriology, Graduate School of Medical Sciences, Nagoya City University, Nagoya, Japan \\ ${ }^{2}$ Department of Clinical Laboratory, Japanese Red Cross Nagoya Daiichi Hospital, Nagoya, Japan \\ ${ }^{3}$ Department of Clinical Laboratory, Japanese Red Cross Nagoya Daini Hospital, Nagoya, Japan \\ ${ }^{4}$ School of Nursing, Sugiyama Jyogakuen University, Nagoya, Japan \\ Email: ^minami@med.nagoya-cu.ac.jp
}

How to cite this paper: Minami, M., Nishiyama, H., Ikegami, S., Hattori, T., Kawashima, M. and Ohta, M. (2018) Analysis of Pyelonephritis-Associated Beta Hemolytic Streptococcus in Japan. Journal of Biosciences and Medicines, 6, 45-52. https://doi.org/10.4236/jbm.2018.612004

Received: October 29, 2018

Accepted: December 2, 2018

Published: December 5, 2018

Copyright $\odot 2018$ by authors and Scientific Research Publishing Inc. This work is licensed under the Creative Commons Attribution International License (CC BY 4.0).

http://creativecommons.org/licenses/by/4.0/

\begin{abstract}
Pyelonephritis is an inflammation of the kidney tissue, calyces, and renal pelvis. It is commonly caused by bacterial infection that has spread up the urinary tract. Common bacteria are Escherichia coli and Enterococcus faecalis. Beta hemolytic streptococci also cause pyelonephritis. Beta hemolytic streptococcus such as Streptococcus agalactiae, and $S$. dysgalactiae subsp. equisimilis, when grown on blood-agar, produce complete hemolysis around each colony, usually cause severe infections including many cases of cellulitis, pneumonia, meningitis and sepsis. However the analysis of pyelonephritis causing beta hemolytic streptococcus has seldom been investigated. In this study, we tried to clarify the relationship between beta hemolytic streptococcus and pyelonephritis patient in Japan. We investigated beta hemolytic streptococcus isolated from pyelonephritis patient at two tertiary care hospitals in the central region of Japan by analyzing the background and antimicrobial susceptibility of beta hemolytic streptococcus. We recovered total sixteen beta hemolytic streptococcus in nine years. In pyelonephritis patients, beta hemolytic streptococcus was isolated from $9 S$. agalactiae and 7 S. dysgalactiae subsp. equisimilis, respectively. The numbers of streptococci from female patients were more than those from male patients. Bacteria were not isolated from under 30 year patients. All $S$. dysgalactiae subsp. equisimilis were isolated from 60 -year-age patients. About one fifth of beta hemolytic streptococcus had erythromycin and minocycline-resistant ability. Four beta hemolytic streptococci that were all $S$. agalactiae, had levofloxacin-resistant ability. Only one $S$. agalactiae had trimethoprim-sulfamethoxazole-resistant ability. Ampicillin was effective against all beta hemolytic streptococci. In our results, beta hemolytic streptococcus caused pyelonephritis in aged people and the quarter of these bacteria had some antibiotics resistance.
\end{abstract}




\section{Keywords}

Beta Hemolytic Streptococcus, Pyelonephritis, S. agalactiae,

S. dysgalactiae subsp. equisimilis, Antimicrobial Susceptibility

\section{Introduction}

Beta hemolytic streptococci are gram-positive bacteria that are mainly involved in $S$. agalactiae, $S$. dysgalactiae subsp. equisimilis, and $S$. pyogenes [1]. As they exist in the part of the commensal flora such as throat, genital and lower gastrointestinal tracts [2], they also cause most of the acute severe infections including many cases of cellulitis, pneumonia, meningitis and sepsis [3] [4]. Especially, they have been recognized as emerging infection in aged people under medical control (mainly malignancy and diabetes mellitus) [5]. This trend might be explained because of increasing number of patients with chronic medical conditions [6]. The increase of drug resistant rate in beta hemolytic streptococcus has also been reported over the last 20 years [7]. The loss of efficacy of erythromycin and clindamycin expressed the increasing rates of resistance in $S$. agalactiae and S. dysgalactiae subsp. equisimilis [3] [4].

Acute pyelonephritis is one of the most common diseases of the kidney. It is a bacterial infection causing inflammation of renal system [8]. Symptoms usually include fever, flank pain, nausea, vomiting, burning on urination, increased frequency, and urgency. The main cause of acute pyelonephritis is Escherichia coli and Enterococcus faecalis [8] [9]. Acute pyelonephritis in the United States is found at a rate of about 16 cases per 10,000 females and about 4 cases per 10,000 males annually [8] [10]. Young sexually active women are the patients that are most often affected by acute pyelonephritis [8]. Despite pyelonephritis improving in most cases, there is still significant morbidity and mortality that can be associated with severe cases of pyelonephritis [8] [11]. Some case reports about beta-hemolytic streptococcus related pyelonephritis demonstrated that pyelonephritis causes pseudohyperaldosteronism, spinal epidural abscess, hemolytic anemia, and acute nephritis [12] [13] [14] [15]. However, the comparative analysis of pyelonephritis by beta hemolytic streptococci has seldom been performed.

The present study was conducted to find out the recent clinical characteristics of pyelonephritis associated beta hemolytic streptococcus isolated at two tertiary care hospitals in the central of Japan.

\section{Materials and Methods}

\subsection{Strains and Clinical Data Collection}

Bacteria were obtained from aseptically specimens at Japanese Red Cross Nagoya Daiichi Hospital and Japanese Red Cross Nagoya Daini Hospital from 2010 to 2018. Japanese Red Cross Nagoya Daiichi Hospital and Japanese Red Cross 
Nagoya Daini Hospital are 852-bed and 812-bed tertiary care hospitals in the central region of Japan, respectively. We used medical records appended to clinical species for the analysis of clinical feature at two tertiary care hospitals. Pyelonephritis was diagnosed by medical doctor such as urologist. It was also involved in bacterial isolation from both blood and urine. We considered several isolates from the same region of the same patient as one isolate per one patient for the analysis in this study. All streptococcus isolates were identified by standard conventional biochemical methods and confirmed by $16 \mathrm{~S}$ ribosomal DNA sequence analysis as described elsewhere [3] [4] [16]. Our experimental design was approved by the ethics committee at both hospitals.

\subsection{Antimicrobial Susceptibility Analysis}

Beta hemolytic Streptococcus isolates were examined for typical five antibiotic susceptibilities as ampicillin, erythromycin, minocycline, levofloxacin, and trimethoprim-sulfamethoxazole. Minimal inhibitory concentrations (MICs) were determined by E-test. Evaluation of susceptibilities was calculated based on Clinical Laboratory Standard Institute (CLSI) breakpoint [17].

\subsection{Statistical Analysis of the Data}

We conducted the statistical analysis with the chi-squared test or Fisher's exact test when appropriate. Differences were considered significant when $p$ was $<0.05$.

\section{Results}

First of all, we evaluated the relationship between pyelonephritis patients' features and beta hemolytic Streptococcus. A total of 16 beta hemolytic Streptococcus ( 9 S. agalactiae and 7 S. dysgalactiae subsp. equisimilis) were isolated. No $S$. pyogenes was found in this study. Three pyelonephritis associated beta hemolytic Streptococcus in 2011, 2013, and 2017 were isolated (Figure 1). But No beta hemolytic Streptococcus in 2013 were isolated. We did not found any significant differences of periods between $S$. agalactiae and $S$. dysgalactiae. Figure 2 showed that the number of female patients was larger than that of male patients, there was no significant differences between genders $(p=0.6567)$. We did not also found any significant differences between $S$. agalactiae and $S$. dysgalactiae subsp. equisimilis. The age range was categorized every 10 years ages in Figure 3. We did not find any patients under 30 year's age (Figure 3 ). The numbers of over 60 - 69 and 80 - 89 year's patients were largest in this study. Only S. dysgalactiae subsp. equisimilis were isolated from 60 - 69 and 80- year's patients. S. agalactiae were isolated at a relatively low age. There were no significant differences between ages $(p=0.3572)$. Finally, we analyzed the antimicrobial susceptibility of beta hemolytic Streptococcus (Figure 4). Ampicillin was susceptible against all beta hemolytic Streptococcus. In our study, total erythromycin and minocycline -resistant rate of beta hemolytic Streptococcus were about 19\% and 25\%, respec- 
tively. Same one $S$. dysgalactiae subsp. equisimilis had both erythromycin and minocycline-resistant ability. Total levofloxacin-resistant rate of beta hemolytic Streptococcus were $25 \%$. All levofloxacin resistant beta hemolytic Streptococcus were $S$. agalactiae. Total trimethoprim-sulfamethoxazole resistant rate of beta hemolytic Streptococcus was about $6.3 \%$. At least one $S$. agalactiae isolates had no susceptible activity against trimethoprim-sulfamethoxazole in this study.

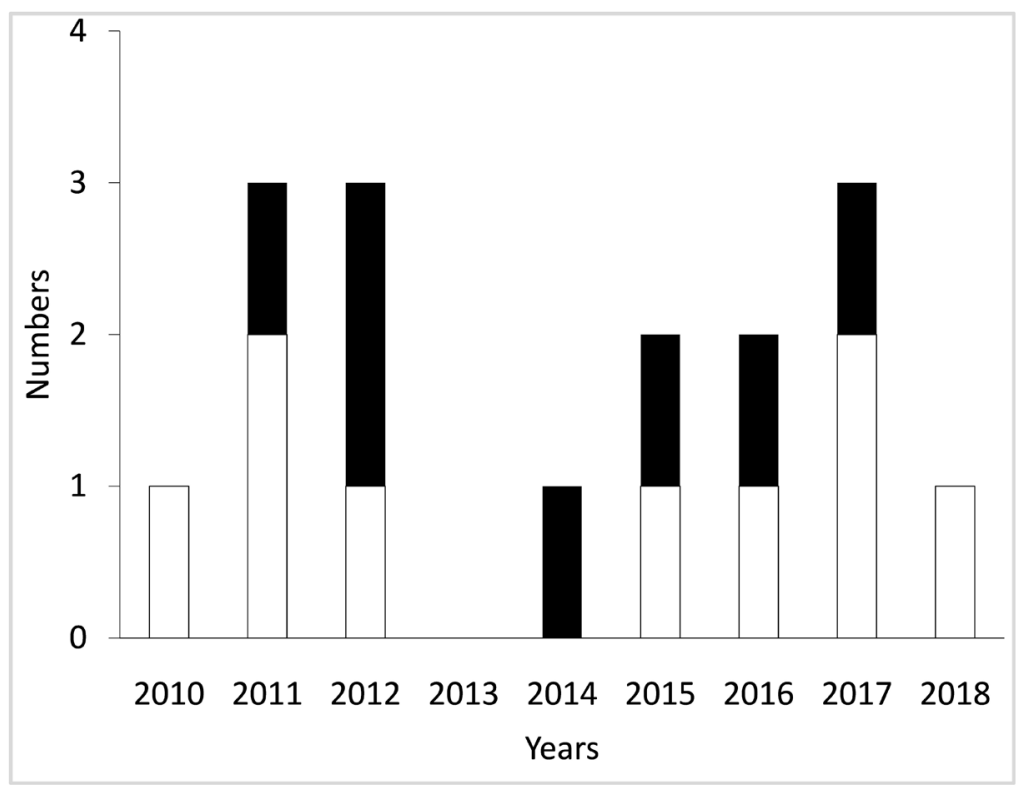

Figure 1. Period distribution of beta hemolytic Streptococcus isolates. White box; $S$. agalactiae. Black box; $S$. dysgalactiae subsp equisimilis.

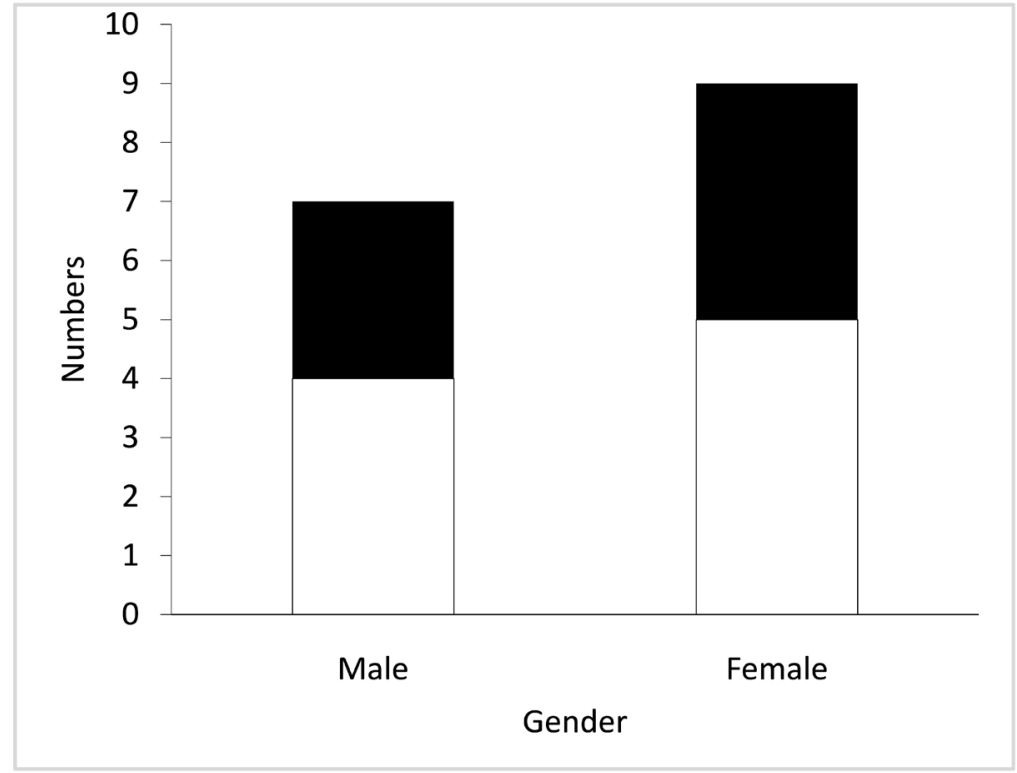

Figure 2. Gender distribution of beta hemolytic Streptococcus isolates. White box; $S$. agalactiae. Black box; $S$. dysgalactiae subsp equisimilis. eriod distribution of beta hemolytic Streptococcus isolates. White box; $S$. agalactiae. Black box; $S$. dysgalactiae subsp equisimilis. 


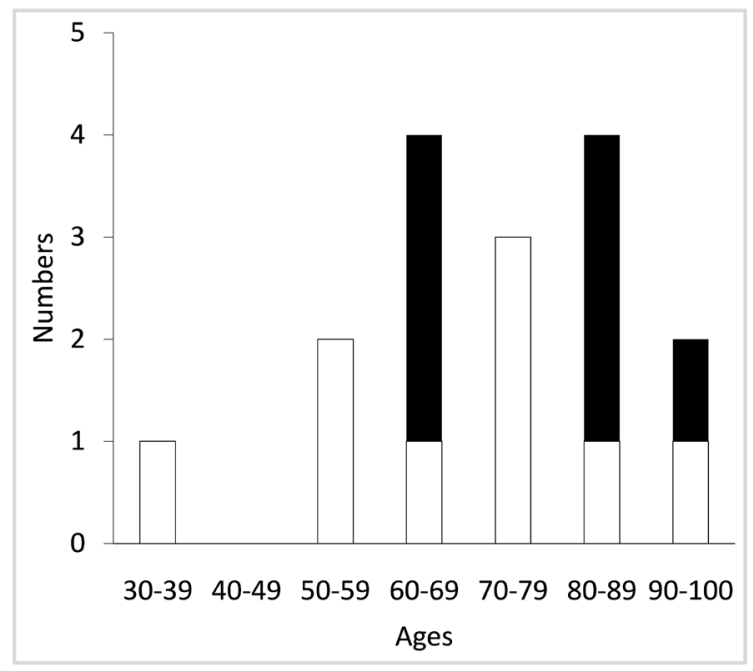

Figure 3. Age distribution of beta hemolytic streptococcus isolates. White box; $S$. agalactiae. Black box; $S$. dysgalactiae subsp. equisimilis.

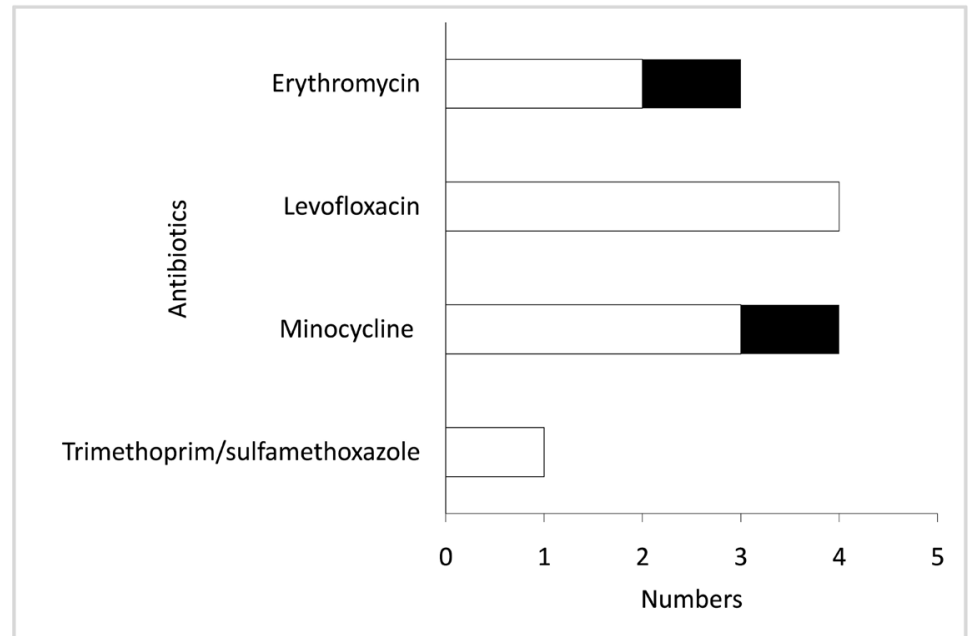

Figure 4. Antimicrobial resistance of beta hemolytic streptococcus isolates. White box; $S$. agalactiae. Black box; $S$. dysgalactiae subsp. equisimilis.

\section{Discussion}

In this study, we described the clinical characteristics and antimicrobial susceptibility patterns of pyelonephritis-associated beta hemolytic Streptococcus isolated from two tertiary care hospitals in the central of Japan among recent 9 years. Although we have little interest of pyelonephritis as compared with other Streptococcal disease before, this study may imply that beta hemolytic Streptococcus will be important pathogen about pyelonephritis. With respect to gender group, the numbers of isolation in female patients were more than those in male patients. This result is almost consistent with previous report [6]. Next, we clarified Streptococcus with age distribution. Although $S$. agalactiae usually causes pyelonephritis in pregnant female, our result showed no pregnant female was involved. As $S$. agalactiae is also popular in neonatal infection, no children oc- 
curred pyelonephritis in our study. Our study demonstrated that beta hemolytic Streptococcus frequently caused pyelonephritis in older people. As we have recognized beta hemolytic Streptococcus as serious infectious disease for female and neonate, we may also reevaluate beta hemolytic Streptococcus as pyelonephritis for aged-people. With respect to antimicrobial susceptibility, we did not find any ampicillin non-susceptible beta hemolytic Streptococcus in this study. Indeed, penicillin resistant $S$. agalactiae and $\mathcal{S}$. dysgalactiae subsp. equisimilis have not been isolated from invasive disease in Japan before [8] [9]. S. agalactiae from female patients with pyelonephritis were resistant to erythromycin and clindamycin [18]. Previous report showed that the macrolide non-susceptible rates of streptococcus was about 30\% [3] [4]. The total erythromycin-resistant rate of beta hemolytic Streptococcus was about $19 \%$ in our study. Previous report demonstrated that the tetracycline resistant rates of beta hemolytic Streptococcus were about 44\% [3] [4]. Our study revealed that minocycline resistant rates of beta hemolytic Streptococcus were $25 \%$. We assumed that antimicrobial resistant rate of erythromycin and minocycline seemed to be slightly lower. Levofloxacin resistant rates of $S$. agalactiae were about $40 \%$ in former study [3] [4] [14] [19]. As our study revealed that levofloxacin resistant rates of beta hemolytic Streptococcus were 25\%, levofloxacin resistant rates of $S$. agalactiae were about $44 \%$. No $S$. dysgalactiae subsp. equisimilis had levofloxacin resistant ability in this study. As trimethoprim-sulfamethoxazole resistant rate of beta hemolytic Streptococcus was about 6\%, trimethoprim-sulfamethoxazole resistant rate of $S$. agalactiae was about $11 \%$ in our study. As fluoroquinolone and co-trimoxazole has been widely used genitourinary disease, digestive disease and respiratory disease [20], careful treatment selection is necessary because levofloxacin and trimethoprim-sulfamethoxazole may be not effective for $S$. agalactiae caused pyelonephritis. Although $S$. dysgalactiae subsp. equisimilis are susceptible of levofloxacin, we assume that the change of fluoroquinolone resistance among beta hemolytic Streptococcus spread worldwide gradually. There are some limitationin this study. As we did not perform genetic analysis of bacteria such as emm typing, we did not clarify the relationship between bacterial virulent genes and clinical features. Although there are few results with significant differences in this study, this seems to be problematic in the small number of samples investigated. From these views, we considered the further necessity of the analysis of beta hemolytic Streptococcus strains from pyelonephritis.

\section{Conclusion}

In summary, we clarified the characteristics of pyelonephritis-associated beta hemolytic streptococcus in two tertiary care hospitals in the central of Japan. Although several antibiotics such as penicillin are effective against beta hemolytic streptococcus, incidence of invasive Streptococcus infection such as pyelonephritis is not significant decreasing. The results from our study suggest the need for further epidemiological surveillance of antibiotic resistant pathogen. 


\section{Acknowledgements}

We thank Mr. Masashi Ishihara and Ms. Miwako Fujimura for special encouragement. This study was supported by a grant-in-aid for research from the Nagoya City University, Japan.

\section{Conflicts of Interest}

The authors declare no conflicts of interest regarding the publication of this paper.

\section{References}

[1] Facklam, R. (2002) What Happened to the Streptococci: Overview of Taxonomic and Nomenclature Changes. Clinical Microbiology Reviews, 15, 613-630.

https://doi.org/10.1128/CMR.15.4.613-630.2002

[2] Dhanoa, A., Karunakaran, R. and Puthucheary, S.D. (2010) Serotype Distribution and Antibiotic Susceptibility of Group B Streptococci in Pregnant Women. Epidemiology and Infection, 138, 979-981. https://doi.org/10.1017/S0950268809991105

[3] Minami, M., Nishiyama, H., Ikegami, S., Hattori, T., Kawashima, M. and Ohta, M. (2016) Trend of Invasive Streptococcus agalactiae at Tertiary Care Hospital in Japan. Journal of Biosciences and Medicines, 4, 15-20. https://doi.org/10.4236/jbm.2016.43003

[4] Minami, M., Sakakibara, R., Imura, T., Watanabe, M., Morita, H., Kanemaki, N. and Ohta, M. (2016) Trend of Respiratory Tract-Associated Streptococcus dysgalactiae Subsp. Equisimilius at General Japanese Hospital from 2013 to 2015. Journal of Biosciences and Medicines, 4, 11-17. https://doi.org/10.4236/jbm.2016.412002

[5] Skoff, T.H., Farley, M.M., Petit, S., Craig, A.S., Schaffner, W., Gershman, K., et al. (2009) Increasing Burden of Invasive Group B Streptococcal Disease in Nonpregnant Adults, 1990-2007. Clinical Infectious Diseases, 49, 85-92. https://doi.org/10.1086/599369

[6] Al Akhrass, F., Abdallah, L., Berger, S., Hanna, R., Reynolds, N., Thompson, S., et al. (2013) Streptococcus agalactiae Toxic Shock-Like Syndrome: Two Case Reports and Review of the Literature. Medicine (Baltimore), 92, 10-14.

https://doi.org/10.1097/MD.0b013e31827dea11

[7] Wang, Y.H., Chen, C.L., Hou, J.N., Wang, Y.R., Lin, T.Y., Wang, M.H., et al. (2015) Serotype Distribution and Resistance Genes Associated with Macrolide and Fluoroquinolone Resistance in Streptococcus agalactiae Isolates from a Hospital in Southern Taiwan. Biomed Journal, 38, 215-220.

https://doi.org/10.4103/2319-4170.138306

[8] Belyayeva, M. and Jeong, J.M. (2018) Pyelonephritis, Acute. StatPearls. StatPearls Publishing, Treasure Island, FL.

[9] Kusama, Y. and Muraki, K. (2018) Bilateral Multifocal Acute Lobar Nephronia Caused by Enterococcus faecalis. BMJ Case Reports, pii: bcr-2018-224336.

[10] Ahmad, S. (2015) Asymptomatic Group B Streptococcal Bacteriuria among Pregnant Women in Saudi Arabia. British Journal of Biomedical Science, 72, 135-139. https://doi.org/10.1080/09674845.2015.11666810

[11] Batista, R.P. and Ferreira, C.R. (2015) Streptococcus agalactiae Septicemia in a Patient with Diabetes and Hepatic Cirrhosis. Autopsy \& Case Reports, 5, 35-43. https://doi.org/10.4322/acr.2015.028 
[12] Kashimada, K., Omori, T., Takizawa, F. and Mizutani, S. (2008) Two Cases of Transient Pseudohypoaldosteronism Due to Group B Streptococcus Pyelonephritis. Journal of Pediatric Nephrology, 23, 1569-1570. https://doi.org/10.1007/s00467-008-0843-9

[13] Chung, S.Y., Chen, C.H. and Yu, W.L. (2005) Spinal Epidural Abscess Caused by Group B Streptococcus in a Diabetic Woman Presenting with Febrile Low Back Pain. Japanese Journal of Infectious Diseases, 58, 177-179.

[14] Vigueras, I., Enríquez, R., Lacueva, J., Arenas, M.D. and Molas, J.R. (1992) Acute Pyelonephritis and Hemolytic Anemia Caused by Streptococcus agalactiae. Revista Clínica Española, 191, 502-503.

[15] Heyman, S.N. and Brezis, M. (1997) Asymptomatic Group-B Streptococcal Pyelonephritis: An Unusual Cause of Acute Renal Failure. Nephron, 75, 243-244. https://doi.org/10.1159/000189542

[16] Hashikawa, S., Iinuma, Y., Furushita, M., Ohkura, T., Nada, T., Torii, K., et al. (2004) Characterization of Group C and G Streptococcal Strains That Cause Streptococcal Toxic Shock Syndrome. Journal of Clinical Microbiology, 42, 186-192. https://doi.org/10.1128/JCM.42.1.186-192.2004

[17] Clinical and Laboratory Standards Institute (CLSI) (2014) Performance Standards for Antimicrobial Susceptibility Testing: 24st Informational Supplement. Clinical and Laboratory Standards Institute M100-S24, Wayne.

[18] Ulett, K.B., Benjamin, W.H., Zhuo, F., Xiao, M., Kong, F., Gilbert, G.L., Schembri, M.A. and Ulett, G.C. (2009) Diversity of Group B Streptococcus Serotypes Causing Urinary Tract Infection in Adults. Journal of Clinical Microbiology, 47, 2055-2060. https://doi.org/10.1128/JCM.00154-09

[19] Ueno, H., Yamamoto, Y., Yamamichi, A., Kikuchi, K., Kobori, S. and Miyazaki, M. (2012) Characterization of Group B Streptococcus Isolated from Women in Saitama City, Japan. Japanese Journal of Infectious Diseases, 65, 516-521. https://doi.org/10.7883/yoken.65.516

[20] Davis, R. and Bryson, H.M. (1994) Levofloxacin. A Review of Its Antibacterial Activity, Pharmacokinetics and Therapeutic Efficacy. Drugs, 47, 677-700.

https://doi.org/10.2165/00003495-199447040-00008 